\title{
IN service specification using the KANNEL language
}

\author{
Kari Granö ${ }^{a}, J^{2}$ rmo Harju ${ }^{b}$, Tapani Järvinen ${ }^{a}$, \\ Tapani Karttunen $^{b}$, Tapani Larikka $^{b}$, Jukka Paakki $^{a}$ \\ ${ }^{a}$ Department of Computer Science and Information Systems, \\ University of Jyväskylä \\ P.O.Box 35, FIN-40351 Jyväskylä, Finland \\ ${ }^{b}$ Department of Information Technology, \\ Lappeenranta University of Technology \\ P.O.Box 20, FIN-53851 Lappeenranta, Finland
}

\begin{abstract}
KANNEL is an application-oriented language for protocol engineering, with integrated support for all the main aspects of implementing typical communications software. Intelligent network services with distributed and interacting components share a number of central features with communications protocols, thus bringing in the possibility of applying protocol engineering techniques within the area of intelligent networking. To verify this view, the KANNEL language is applied to the specification of an intelligent calling card service. The specification includes both visual and textual notations of KANNEL.
\end{abstract}

\section{INTRODUCTION}

Recent active research on application-oriented languages promotes the view that application areas with specialized nature, such as protocol engineering, should be supported with dedicated tools founded on high-level concepts that match with the central characteristics of the applications. This view is in sharp contrast with the simplistic approach of engineering every application using general-purpose languages, such as $\mathrm{C}$.

KANNEL [GHP94] is an application-oriented language designed especially for protocol engineering. While the design principle behind most of the conventional languages in this area, such as Estelle (Extended State-Transition Language) [BuD87], LOTOS (Language of Temporal Ordering Specification) [BoB87], SDL (Specification and Description Language) [BeH89], ASN.1 (Abstract Syntax Notation One) [Ste90], and TTCN (Tree and Tabular Combined Notation) [ISO89], has been on providing support for solving only some narrow 
problems within protocol engineering, KANNEL aims at covering the whole discipline in a uniform manner. In this way the need for using several different languages and several unrelated software tools to implement a protocol disappears since the whole task can now be managed under a single language and its integrated environment. The most notable features of KANNEL to support integrated protocol engineering are hierarchical finite state machines, distribution, interfaced layering, and encoding/decoding facilities.

While the main application area of KANNEL is protocol engineering, it can well be used as the implementation language of general (distributed) software systems. This more versatile side of KANNEL calls for more general software engineering capabilities which include objectorientation, visual design facilities, and an integrated tool set with a graphical user interface.

Intelligent network (IN) services are by their nature specialized distributed applications with the service specific logic residing in SCP, the service control point. The functionality of the service is realized in close co-operation with SSP, the service switching point, which is an integral part of a modern telephone exchange. In their interactions, SCP and SSP follow the standardized protocols described e.g. in ETSI's CS-1 standard [ETS94]. Thus, there are similar elements in engineering of IN services and in typical protocol engineering problems, which brings in the possibility to apply KANNEL in the specification of IN services.

In this paper we apply the KANNEL language to specifying a calling card service that allows telephone users to use any telephone for outgoing calls, charged to their own account [Kar94]. Authentication is normally based on a personal access number together with a personal identification number (PIN code) but a set of trusted A-subscriber numbers can be provided, authorized without PIN code. Moreover, the user can change his/her PIN code when using the service. The service also provides for specially priced short numbers, screening of unauthorized numbers, and special numbers with configurable destination. We describe the KANNEL specification of the calling card service, concentrating on the central SCP component.

Intelligent network services are tightly coupled with the existence of modern telephone exchanges (even though a well defined IN service is independent of the implementation of the underlying switching network). Since KANNEL does not directly support low-level physical aspects of telecommunications, it can be argued that KANNEL is not a full-fledged implementation language for IN applications. To overcome such obvious problems, we do not completely specify the SSP component of the calling card service but instead incorporate it into the KANNEL specification as an external entity, assumed to be implemented by some suitable means. This also holds for the database module of the service (SDP), due to the lack of decent database facilities in KANNEL. However, the communication protocol between all the main components of the service (SSP, SCP, SDP) is fully included in the specification.

We proceed as follows. The KANNEL language and its programming environment are summarized in Section 2. The personal call service and its specification in KANNEL are presented in Section 3. Finally, conclusions are drawn in Section 4. The service specification in textual KANNEL is included as an Appendix.

\section{THE KANNEL LANGUAGE AND ENVIRONMENT}

Starting from the development of packet networks and telephone exchanges with stored control, the role of software has become more and more important in communication systems. This is largely due to the increased intelligence built into distributed systems to facilitate the 
supply of versatile services through communication networks. Protocols are the key elements in providing this intelligence, and during the last twenty five years we have seen a tremendous rise in the scope and complexity of protocols on various levels of communication architecture.

Protocol engineering may well be the most complex field of software engineering where (semi-)automatic implementation based on formal techniques has been set as a realistic goal. There are five well-known languages that have been designed to formally specify distributed systems, and to generate communication software from specifications.

While there has been extensive research and development efforts on protocol design, the proposed languages usually have a restricted focus of application. For instance, Estelle and SDL can be used for defining the internal control behavior of a protocol entity (as an extended finite state machine) with a very abstract notion of data, and ASN.1 is intended solely for describing the conveyed data units without specifying any operational characteristics of the protocols. LOTOS, in turn, is too abstract for being implemented efficiently with current technology.

A survey of protocol tools used by Finnish industry is given in [AHG93]. The conducted experiments clearly show that the low quality and limited scope of the applied languages and the inconsistency of the software tools based on them make practical protocol work overly laborous. The essential conclusion about the current state-of-the-art is that although all aspects of protocol engineering (or, more generally, distributed communicating systems) can be handled using current languages and tools, the task is rather hard to carry out systematically and consistently.

The KANNEL language is an evolving attempt to overcome the shortcomings mentioned above. In protocol engineering, the usual approach has been to separate the design of a protocol from its implementation, and to consider functional logic and concrete data units as orthogonal issues. We believe that a unified scheme which would provide tools for all phases of protocol engineering is a desirable goal. To reach this, KANNEL integrates special protocol engineering facilities with general software engineering principles.

The key aspects of KANNEL that are related to software engineering in general are briefly described below.

\section{object-orientation:}

KANNEL supports the class and object concepts of object-oriented languages. There are two fundamental object categories in KANNEL: value objects are always represented by value, while reference objects are represented by reference. This division is a compromise between efficiency and orthogonality - representing all objects via references would be inefficient, and it is desirable to have the primitive types, such as integer, part of the class system.

In addition, objects may be local or distributed; this distinction affects the way the object can be communicated with. ${ }^{1}$ Local objects are flexible to use, but do not fulfill strong distribution semantics.

KANNEL has adopted its notion of classes most notably from the Sather language [Omo93], in particular the facility of incremental superclassing and the explicit separation of (multiple) inheritance from code reuse.

\footnotetext{
${ }^{1}$ KANNEL does not offer a completely transparent distribution in the manner of e.g. Emerald [Jul88]. The reasons for this lie in efficiency and application area.
} 


\section{interfaces:}

An interface class is used to define the abstract service interfaces of sets of classes. An interface class has no implementation (in this sense it resembles the deferred class concept of Eiffel [Mey88]). Subtyping in KANNEL is based on interface classes.

\section{code reuse:}

KANNEL allows a class to reuse the implementation of another class. Code reuse has no effect on type compatibility; it can be simply regarded as textual inclusion. This reflects the opinion that subtyping and code reuse are two distinct things that should be kept separate [Ame89].

\section{visuality:}

The visual components of KANNEL support the design of behavioral components of a protocol. It has been argued that a visual notation can greatly enhance the understandability of complex constructs [Har88]. In particular, this seems to be true for state machines.

\section{integration:}

Because KANNEL can be used to describe all the relevant aspects of a protocol, the supporting tools can be integrated. This implies that we do not need to generate code by several distinct tools and then integrate the code fragments with hand-written code. Instead the code generation process is fully automated in the sense that the resulting code need not be complemented.

\section{maintenance:}

The KANNEL environment supports multiple specifications that represent the system on different levels of abstraction. The integrity of these specifications is preserverd by the environment and changes to one specification are automatically propagated throughout the system.

Beside these general software engineering aspects, KANNEL provides for the following dedicated facilities of protocol engineering:

\section{distribution:}

In KANNEL, the service interfaces provided by a distributed class are separated from their implementation into a channel. This decision has the following advantages:

- Separate compilation is facilitated. The monolithic class mechanism employed in e.g. C++ implies that even changing the private part of a class requires recompilation for all clients. By separating these concerns (and following the tradition of Ada, Turbo Pascal, Modula-2, etc.) more flexibility is gained.

- The unidirectional nature of messages is supported better. By giving different views into the service, related messages going in different directions may be syntactically attached together. This leads to a more concise representation of the mutual relationship between a client and its server.

- When channel is made an explicit language construct, we may better grasp the notion of transfer syntax.

- The view concept provides static security and more readable specifications. 
A channel is a variant of an interface class that specifies a multiway relationship for a set of distributed classes. Thus, the channel specifies the abstract service employed between a set of (typically two) peers. ${ }^{2}$ The service is split into a set of disjoint views [KMR92], each grouping a set of messages that travel in the same direction. A channel may also specify the transfer syntax used to represent the concrete content of the messages.

\section{communication:}

In KANNEL, a transfer syntax is a class definable by the protocol writer. Each transfer syntax specifies a method of encoding/decoding all values of both basic and compound types. Functions for encoding and decoding data types are automatically created and applied when needed.

The idea of transfer syntax in KANNEL is similar to that in ASN.1. However, the realization of this idea is more high-level and advanced as a language.

\section{concurrency:}

KANNEL supports concurrency in terms of active objects. At most one thread of execution is active within each active object at a time. This approach, although excluding e.g. concurrent read access, provides a simple and safe solution to mutual exclusion problems.

\section{functional logic:}

KANNEL has adopted the statechart formalism [Har87] with some modifications as the means to describe the concurrent semantics of an active object. A statechart specifies the exact set of messages the object will respond to in a given state and thus maintains its integrity --- this can be viewed as stating as a precondition those messages that are valid for a given state. The communication model is asynchronous so as to offer greater efficiency and flexibility in communication operations.

\section{synchronization:}

As pointed out above, only one thread can be executing within an active object at a time. This implies that we need not consider synchronization within an active object.

\section{layers:}

Communication protocols are conventionally organized as layers. A layer (when both parties are concerned) consists of peer processes and the interconnecting channel. KANNEL employs static groups to create a layer by interconnecting the peer processes with a channel. To support the common practice of layered communication, the layers can be refined into more concrete ones (that is, a mapping from a layer into its lower neighbour can be systematically engineered).

\section{reactivity:}

Protocol engines are reactive in the sense that their operation is directed by incoming messages that typically make some request. Depending on the message, the engine may respond to it by, for example, sending some other message to another engine. The reactivity properties of active

\footnotetext{
${ }^{2}$ This is a more general concept than "client-server", as it recognizes the fact that these roles are usually viewdependent, i.e. that a server is also a client (for someone else).
} 
objects are defined by statecharts. They define the set of incoming and outgoing messages, and the way each incoming message is responded to.

While the intended application area of KANNEL is protocol engineering, its key facilities are quite usable in other branches of software engineering as well, for instance in the implementation of distributed systems or intelligent networks. This more general side of KANNEL will be demonstrated in the next section where the language is applied to specifying an intelligent calling card service. The specification also serves as an example of a KANNEL program. Note, however, that while KANNEL as a complete programming language can be used to implement any (computable) system, it does not necessarily provide the proper level of abstraction in special areas other than protocol engineering.

We are currently implementing a protocol engineering environment that supports the KANNEL language [JGH94]. Our environment aims at integrating all the central tools needed in protocol engineering, thus making it possible to specify a protocol and to generate the corresponding implementation with a single tool set.

The main component of the environment is a visual KANNEL editor which is the default tool for writing a protocol specification. The specifications written with the visual editor are translated into the purely textual form of KANNEL which can also be used directly as the specification language. A compiler will translate the (user-written or internally produced) textual form into some general purpose programming language. Our intention is also to implement transformators between KANNEL and current protocol languages, so as to support interfacing with existing protocol standards and specifications. A long-term plan is to provide graphical analyzers and simulators directly on the visual KANNEL level. The tool set will be running under a common graphical user interface.

\section{PERSONAL CALL SERVICE}

\subsection{Service description}

The personal call service consists of two functional entities. These entities are access/authentication and placing outcall/PIN change.These two entities can be further divided into eight functional parts.

A user accesses and identifies him/herself to the system by entering a user specific access number. There are two ways of authentication. The first one is to ask the user to enter his/her personal identification number (PIN). The number of retries in case of a false combination of PIN and access number is limited. In the case of a false combination, the user is prompted to enter again both the access number and the PIN. When the maximum number of retries is exceeded, the user is notified and the call is terminated. This also blocks the service from the user. When the user has entered the required information correctly, he/she is asked to make an outgoing call or to select the PIN change procedure. The PIN can be either of fixed or of variable length. The other way of authentication is to check for an A-subscriber number. A user can have a limited number of A-subscriber numbers for which a PIN is not required.

The outgoing calls are checked for authentication of the particular user. If the entered number is allowed for this user, the call is completed; otherwise the call is abandoned. The verification is made in terms of global and user specific screening lists. There are two different types of outgoing numbers: short numbers of one or two digits, and special service and 
network numbers of at least three digits. When the user enters a number, the system checks from a database that a physical correspondence exists. If a short number or a special service number has been entered, the system gets the actual physical number from the database and connects the call; otherwise the call is simply connected to the entered number (if allowed).

After invoking the PIN change procedure, the user is asked to enter his/her old PIN. If the entered PIN matches the current PIN, the user is asked to enter a new PIN. The new PIN is checked for validity (e.g., that it is not 1111). If the new PIN is invalid, the operation is abandoned. If the entered new PIN is valid, the system asks the user to re-enter it. If the reentered PIN matches the previously entered one, the database is updated; otherwise the old PIN remains valid.

After any completed operation the user is prompted for another operation. This is the so called global follow-on feature.

At any state of the whole process, the user can close the personal call service by hanging up the phone.

\subsection{Service specification in KANNEL}

In this section we specify the personal call service described above with the KANNEL language. The presentation is mostly graphical but fragments of textual code are given when a more elaborate presentation is appropriate. A complete textual presentation can be found in the Appendix.

\subsubsection{General architecture}

The system is composed of three main components (see Figure 1):

ssp (service switching point) is in essence the user interface to the system.

scp (service control point) is where the intelligence resides and, as such, the main subject of our interest. scp interacts both with ssp and sdp.

sdp (service data point) is the database which holds the information about user identification and authentication, short numbers, number screening, etc.

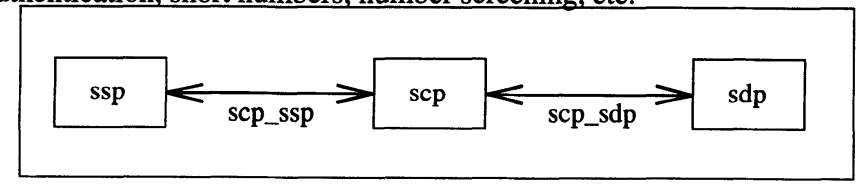

Figure 1. General architecture

Of these, scp is our main target, whereas ssp and sdp are merely supporting entities for which we provide only an abstract description. In Figure 1 the outermost block corresponds to the group concept of KANNEL. The inner blocks correspond to classes, and the bidirectional arrows denote channels. 


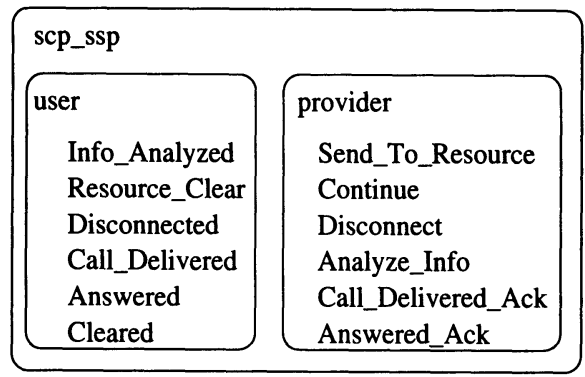

Figure 2. Structure of the scp_ssp channel.

To specify how scp interacts with ssp and sdp, we fix the structure of the channels scp_ssp and scp_sdp. As shown in Figure 2, the channel scp_ssp provides two views, namely user and provider. The user view conveys messages (Info_Analyzed, etc.) from ssp to scp. The provider view, in turn, conveys messages (Send_To_Resource, etc.) from scp to ssp. Similarly, Figure 3 illustrates the structure of the channel scp_sdp.

For illustrating how a message is specified in KANNEL, let us have a look at Query_Result for the user view of the scp_sdp channel:

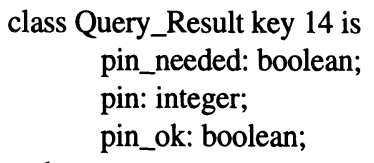

This message has three parameters, the boolean-valued pin_needed and pin_ok, and the integer-valued pin. The message has a key value 14 which is used to encode it uniquely over a transfer syntax. The message system of KANNEL resembles the approach taken in the data description language ASN.1 [Ste90] with a facility of automatic encoding and decoding under a complete transfer syntax. We omit a further elaboration in this case, and assume that the underlying transfer syntax is one of the standard ones, akin e.g. to the basic encoding rules (BER) of ASN.1.

Having the general framework defined, we can now move on to refine the internal structure of the service control point, scp.

user
Query_Result
Update_Confirmation

Figure 3. Structure of the scp_sdp channel. 


\subsubsection{Service control point}

The service control point mainly takes care of controlling and informing ssp, by using the services offered by sdp. The duty of scp is largely to receive messages from ssp and sdp, and to send appropriate replies back to them.

Figure 4 sketches the complete visual specification of the personal call service. It has the same basic elements as Figure 1. However, now the specification of scp is much more detailed. The state machine of scp consists of two main states, login and outcall_PIN_change. User identification and authentication are handled in state login, while an outgoing call and a PIN code change are handled in state outcall_PIN_change.

State login is composed of five substates of which idle is the default, i.e. the state entered whenever login is entered. State idle has one transition labelled Info_Analyzed/Query. The interpretation of this label is to fire the transition whenever the signal (or message) Info_Analyzed is received. Upon reception of the signal, the message Query is emitted. Finally, control moves to state query.

State query has two transitions, labelled Query_Result(PIN not needed) / e and Query_Result(PIN needed) / Send_To_Resource("Provide PIN") (here e stands for an empty action). Considering the signal names only, this may seem to make the machine nondeterministic. However, taking a look at the corresponding textual code fragment for state query, we see how this is solved:

state query arcs

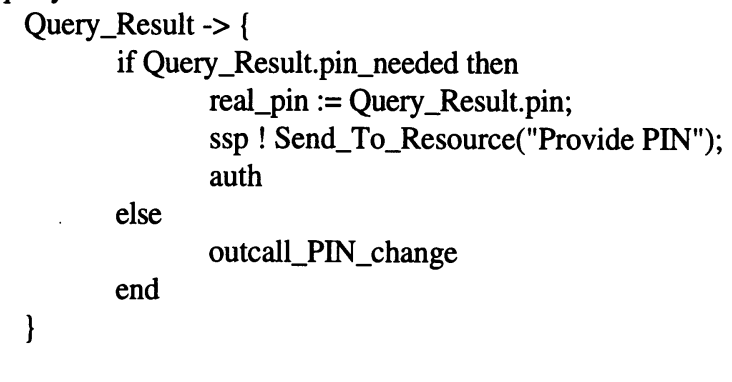

Here Query_Result is the triggering message, and the associated action is enclosed in curly braces. The destination state is either auth or outcall_PIN_change depending on the Boolean value of Query_Result.pin_needed. 
In other words, on a single incoming message we can make further decisions about the following actions according to e.g. parameters of that message. In this case we check the

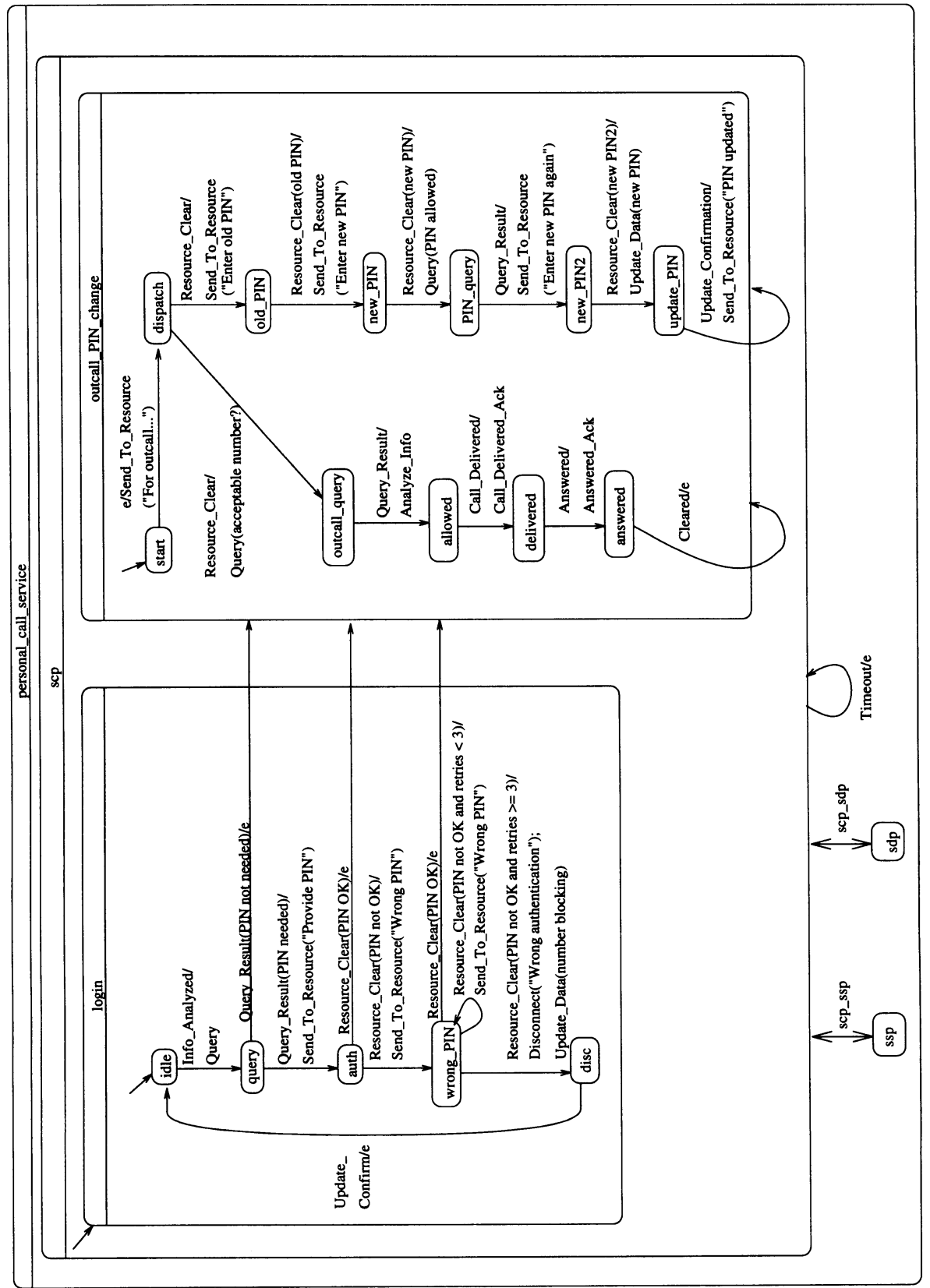

Figure 4. Visual specification of the personal call service. 
pin_needed parameter and decide whether or not the user has to supply a PIN.

If a personal call service user calls from a known telephone machine, he/she does not need to supply a PIN code. Otherwise a PIN is requested from the user who may retry for three times. If the user fails to provide a correct PIN code three times in a row, his/her personal call access number is blocked.

Another substate of scp is outcall_PIN_change. This state deals with outcalls and the PIN change procedure. It has 11 substates, of which start is the default. The user selects either one of the two services by entering a destination number or the \#-symbol. In substate dispatch we choose the correct path to accomplish the selected service.

If the user selects the outcall service, the machine moves to state outcall_query. Here scp makes a query to sdp to check whether the user is allowed to place an outcall on the indicated number, and whether it is a special number or a short number.

In case the user selects the PIN change procedure, he/she is prompted for the old PIN. If he/she provides the correct PIN, the new PIN shall be entered. If the new PIN is acceptable (i.e., it is a combination of digits which is not too easy to guess), the user is prompted to provide the new PIN again. If the entered PINs match, the database at sdp is updated by the new PIN.

\subsubsection{Service switching point and service data point}

From the viewpoint of the service control point (scp), the service switching point (ssp) and the service data point (sdp) are abstract, supporting entities which can be seen as black boxes. For this reason we provide only a high-level specification for them, demonstrating the abstraction capabilities of KANNEL. In Figure 4, ssp and sdp are just plain boxes, connected to scp with channels. In the textual form of KANNEL this is specified by prefixing the class definition by the keyword external:

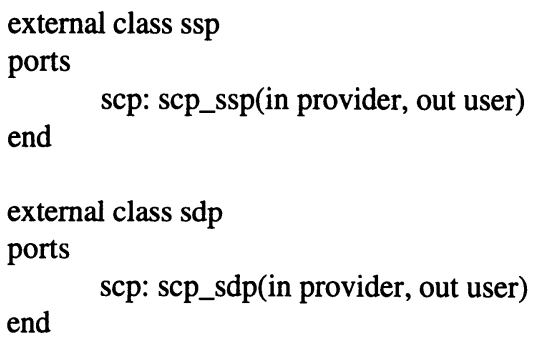

As these classes are external, we do not specify their functional behavior. All we need to know about them is the interface, which is specified by providing the ports clause. As usual, the external entities (here: ssp and sdp) are assumed to have a complete definition or implementation whose details, however, are irrelevant for the main subject (here: scp).

\subsubsection{The complete system}

We conclude the building of the personal call service by collecting together all the components presented above. The visually specified parts of the complete system are shown in Figure 4 except for the channel specifications which are given in Figures 2 and 3. The group combining ssp, scp, sdp, and the channels is textually defined as follows: 
group personal_call_service

ports

local

$$
\begin{aligned}
& \text { r: ssp; } \\
& \text { s: scp; } \\
& \text { t: sdp; }
\end{aligned}
$$

is

s.ssp.attach(r.scp);

s.sdp.attach(t.scp);

end personal_call_service

The textual form makes use of port names that correspond to the endpoints of channels in Figure 4. The channel structure and the distributed object structure of the system are static, that is, this part of the system configuration cannot be adjusted while running the system.

While the presented KANNEL solution to the personal call service can be regarded appropriate for a specification, it falls somewhat short for a running implementation. In particular, a complete implementation should pay attention to the possibility of abnormal cases, e.g. reception of unknown (or damaged) messages by the scp, or reception of nothing while scp is actively executing its state machine. Also the user interaction with the ssp should be checked for correctness. For timeout situations (see Figure 4) there would be a special timer system (a KANNEL class), and for the uninterpretable communication situations the implementation would be prepared for "any" input matching none of the accepted messages. For reasons of space, these abnormal cases are not handled in our specification.

\section{CONCLUSIONS}

We have applied the protocol engineering language KANNEL to the specification of an intelligent calling card service. Due to the special nature of KANNEL, we have concentrated on those aspects of the service that are most protocol oriented: the interaction between the main components of the system, the service switching point (ssp), the service control point (scp), and the service data point (sdp).

The main functionality of the application resides at the scp. Hence, our specification is also founded on the description of scp's functional logic. In our opinion, the protocol engineering paradigm pursued by the KANNEL language proves to be quite suitable at least for this aspect of the IN concept. We have described the secondary components of the system, ssp and sdp, as external entities whose details are not given in KANNEL. Note, however, that it would be quite possible to completely specify ssp and sdp in KANNEL as well, even though the abstraction level needed by their special characteristics would probably not be sufficiently covered by KANNEL. This observation can be tuned to a view of future development of the language: To more properly support the implementation of IN applications, KANNEL should provide dedicated facilities for describing databases (in this case: sdp) as well as (abstract) user interfaces (in this case: ssp).

The PROFILE project (PROtocol Facilities in an Integrated Language and Environment) on designing and implementing the KANNEL language has been running since the end of 1992. The prestudy phase and the language design phase of the project have been finished, and we are currently in the implementation phase, working especially on the visual KANNEL editor. 
An initial implementation will be finished during 1995, after which a final industrialization phase has been planned to take place.

\section{ACKNOWLEDGEMENTS}

The PROFILE project is mainly funded by the Technology Development Centre of Finland (TEKES). The project is supported by an industrial steering group consisting of Ari Ahtiainen (Nokia Research Center), Tom Leskinen (Nokia Cellular Systems), Olli Martikainen (Telecom Finland), Heikki Oukka (X-Net), Jari Rautiainen (Nokia Telecommunications), Jari Simolin (Nokia Telecommunications), and Asko Vilavaara (Technical Research Center of Finland). The ideas and suggestions of the steering group are gratefully acknowledged. The working environment has been provided by Nokia Research Center.

\section{REFERENCES}

[AHG93] Arvonen K., Harju J., Granö K., Paakki J.: A Survey of Software Tools in Protocol Engineering. Report 1, Department of Information Technology, Lappeenranta University of Technology, 1993.

[Ame89] America P.: Issues in the Design of a Parallel Object-Oriented Language. Formal Aspects of Computing 1, 1989, 366-411.

[BeH89] Belina F., Hogrefe D.: The CCITT Specification and Description Language SDL. Computer Networks and ISDN Systems 16, 4, 1989, 311-341.

[BoB87] Bolognesi T., Brinksma E.: Introduction to the ISO Specification Language LOTOS. Computer Networks and ISDN Systems 14, 1, 1987, 25-59.

[BuD87] Budkowski S., Dembinski P.: An Introduction to ESTELLE: A Specification Language for Distributed Systems. Computer Networks and ISDN Systems 14, 1, 1987, 3-23.

[ETS94] ETSI (European Telecommunications Standards Institute): Intelligent Network (IN); Intelligent Network Capability Set 1 (CS 1), Core Intelligent Network Application Protocol (INAP): Part 1: Protocol specification. Draft PrETS 300 374-1, 1994.

[GHP94] Granö K., Harju J., Paakki J., Järvinen T.: Proposal for a Protocol Engineering Language. Technical Reports TR-6, Department of Computer Science and Information Systems, University of Jyväskylä, 1994.

[Har87] Harel D.: Statecharts: A Visual Approach to Complex Systems. Science of Computer Programming 8, 1987, 231-274.

[Har88] Harel D.: On Visual Formalisms. Communications of the ACM 31, 5, 1988, 514530.

[ISO89] ISO (International Organization for Standardization): The Tree and Tabular Combined Notation (TTCN). ISO/IEC DIS 9646-3, Information Technology OSI Conformance Testing Methodology and Framework, Part 3, 1989.

[JGH94] Järvinen T., Granö K., Harju J., Paakki J.: An Integrated Environment for Protocol Engineering. In: Proc. Nordic Workshop on Programming Environment Research (NWPER'94) (B.Magnusson, G.Hedin, S.Minör, eds.), Lund, 1994. Report LUCS-TR: 94-127, Department of Computer Science, Lund University, 1994, 177193. 
[Jul88] Jul E. et al.: Fine-grained Mobility in the Emerald System. ACM Transactions on Computer Systems 1, 6, 1988, 109-133.

[Kar94] Karttunen T.: Personal Call Service Description. Draft, Telecom Finland, 1994.

[KMR92] Koivisto J., Malka J., Reilly J.: Object-Oriented Implementation of Telecommunication Software Using the OTSO Enviroment. In: Proc. IFIP TC6 4th Conf. on Information Networks and Data Communication (INDC-92), Espoo, Finland, 1992, 293-304.

[Mey88] Meyer B.: Object-Oriented Software Construction. Prentice-Hall, 1988.

[Omo93] Omohundro S.: The Sather 1.0 Specification. Unpublished; available electronically as file manual-1.0v5.ps. $\mathrm{Z}$ in directory /pub/sather via anonymous ftp to ftp.icsi.berkeley.edu.

[Ste90] Steedman D.: ASN.1 - Tutorial \\& Reference. Technology Appraisals Ltd., 1990. 


\section{APPENDIX TO ARTICLE "IN SERVICE SPECIFICATION USING THE KANNEL LANGUAGE":}

\section{TEXTUAL KANNEL SPECIFICATION OF THE PERSONAL CALL SERVICE}

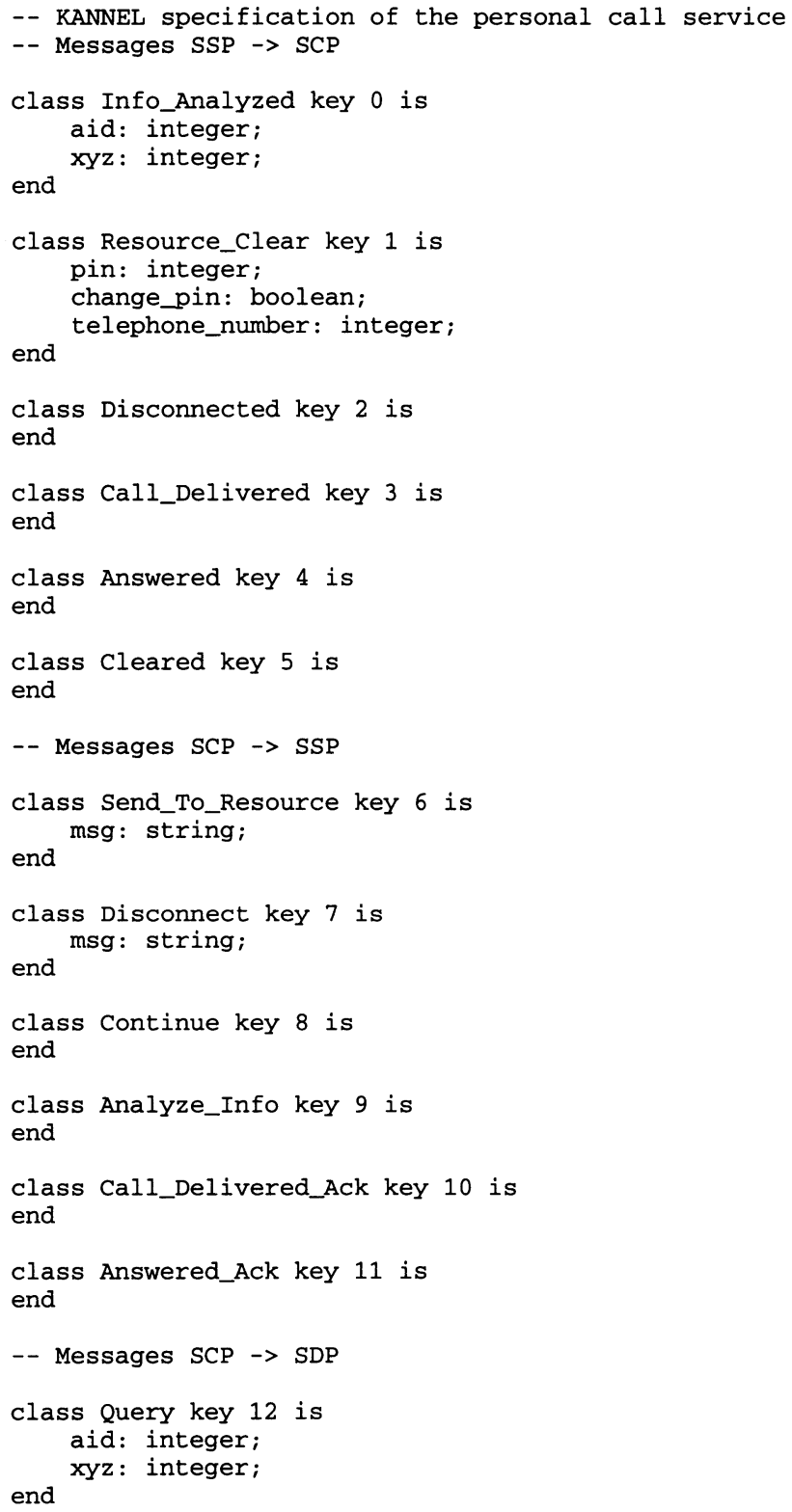




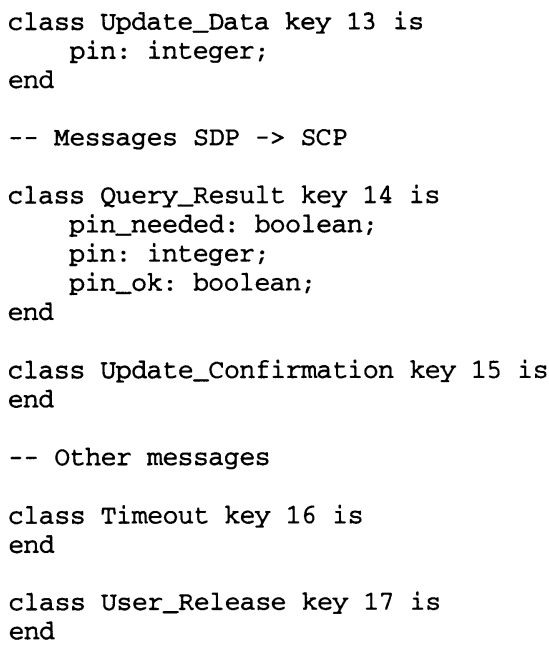




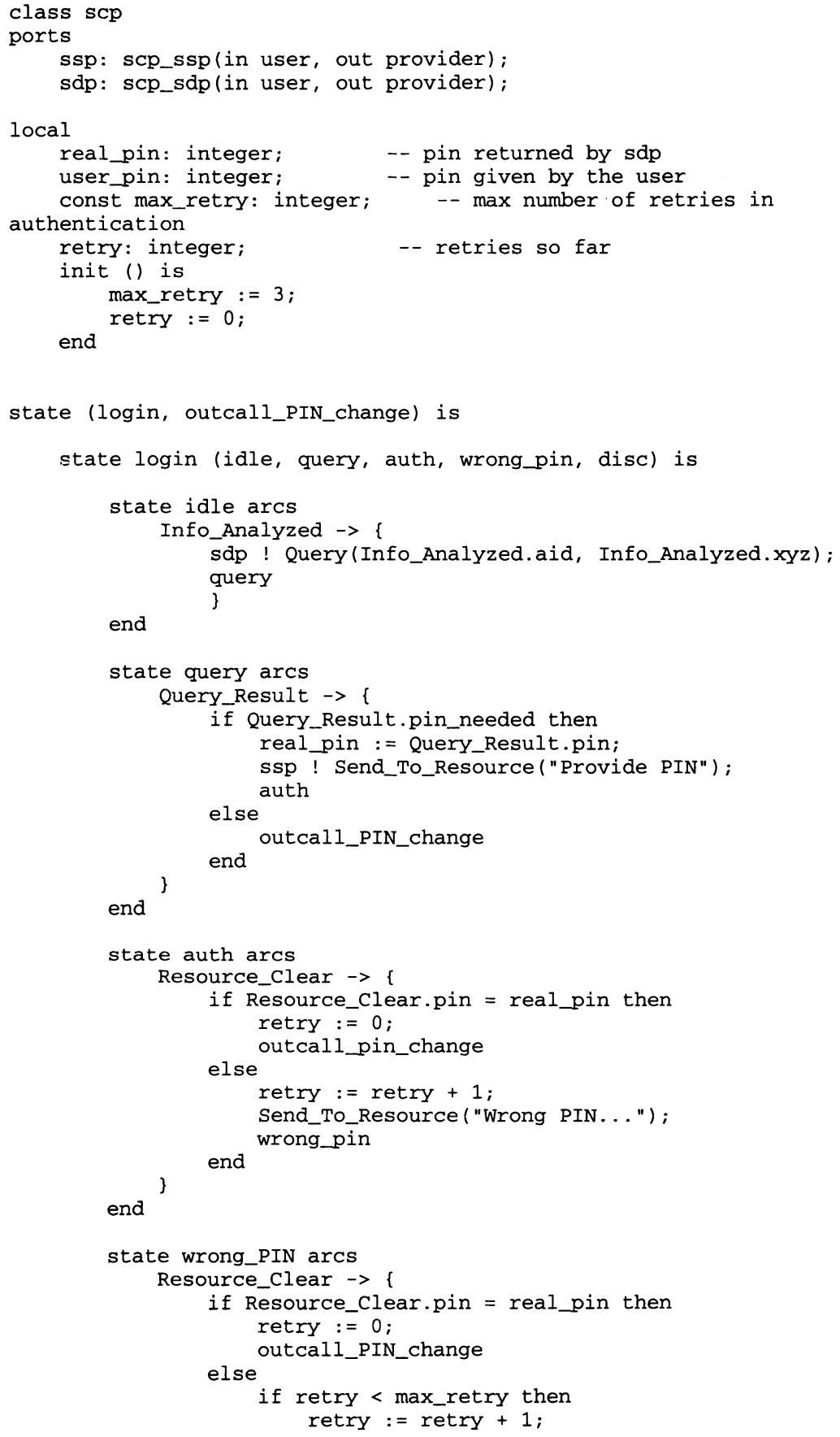




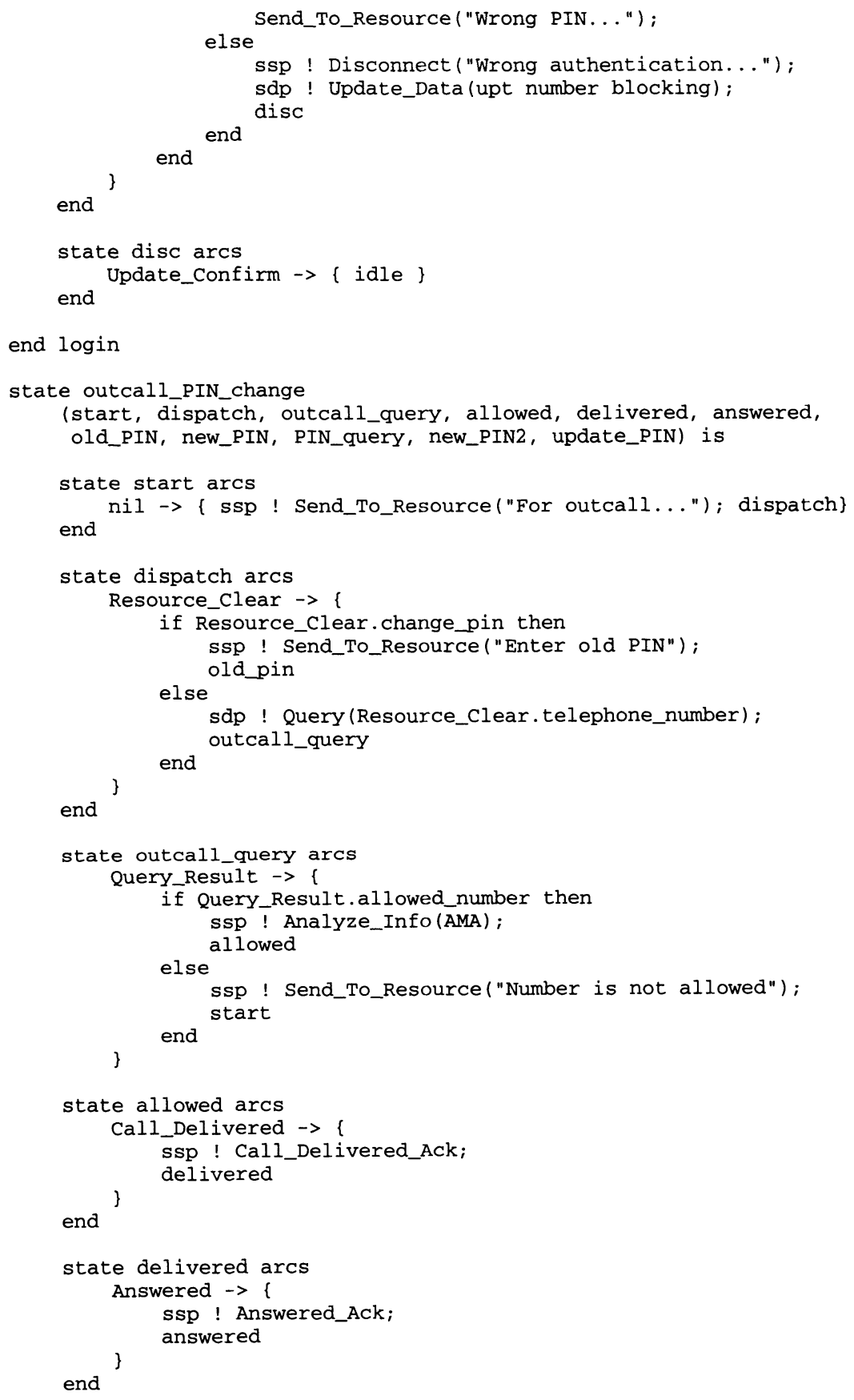




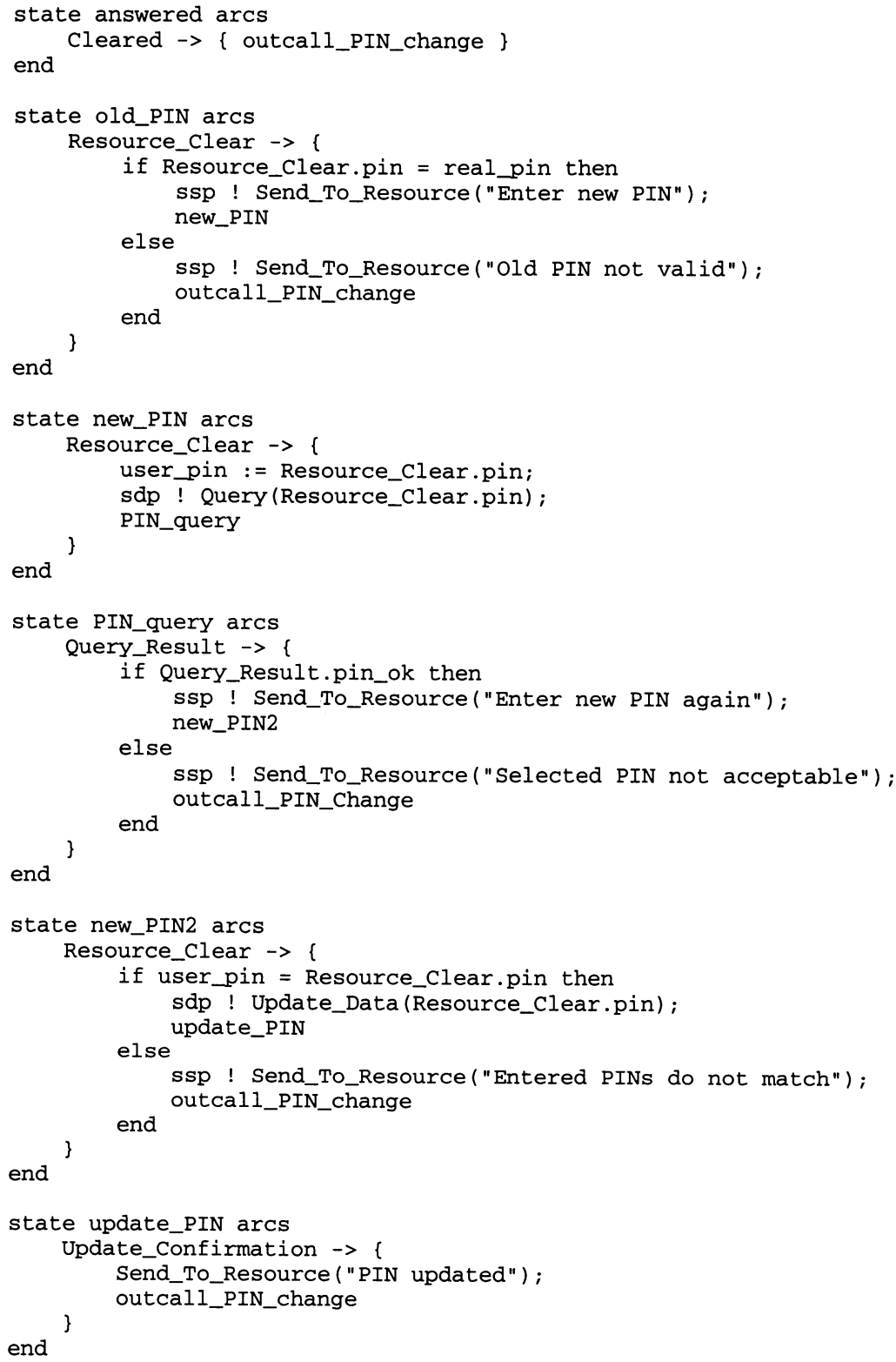




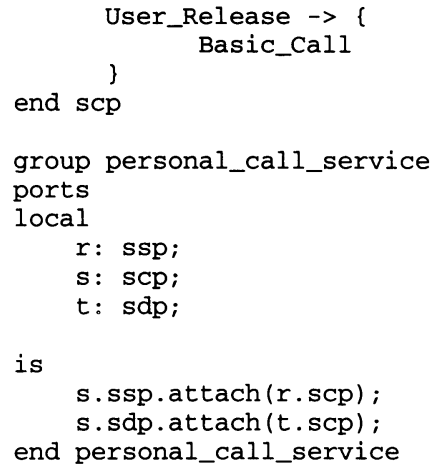

